DOI: $10.15393 /$ j3.art.2019.6690

UDC 517.537

K. G. Malyutin, A. L. Gusev

\title{
GEOMETRIC MEANING OF THE INTERPOLATION CONDITIONS IN THE CLASS OF FUNCTIONS OF FINITE ORDER IN THE HALF-PLANE
}

\begin{abstract}
The aim of this paper is to study the interpolation problem in the spaces of analytical functions of finite order $\rho>1$ in the half-plane. The necessary and sufficient conditions for its solvability are found in terms of the measure defined by the nodes of interpolation.
\end{abstract}

Key words: half-plane, function of finite order, free interpolation, Nevanlinna measure, interpolation sequence

2010 Mathematical Subject Classification: 30E05

1. Introduction, Definitions, and Notations. Interpolation problem is called simple free interpolation problem if multiplicities of the interpolation nodes are equal to unity and restrictions on the values of interpolation function $F$ at these nodes are necessary restrictions related to the fact that the function $F$ must to belong to the considered space. In [6], the problem of simple free interpolation in the spaces of analytical functions of finite order $\rho>1$ in the half-plane $\mathbb{C}_{+}=\{z: \operatorname{Im} z>0\}$ was considered. In this article, we use the definitions and notation of [6]. Denote by $[\rho, \infty]^{+}$the space of analytical functions of finite order $\rho>1$ in $\mathbb{C}_{+}\left[2\right.$, Chapter I, §1]. Let $A=\left\{a_{n}\right\}_{n=1}^{\infty} \subset \mathbb{C}_{+}$be a sequence of distinct complex numbers such that all limit points of $A$ are on the real axis and infinity.

Definition 1. A sequence $A$ is called an interpolation sequence in the space $[\rho, \infty]^{+}$if for any sequence of complex numbers $\left\{b_{n}\right\}_{n=1}^{\infty}$ satisfying the conditions

$$
\sup _{n \in \mathbb{N}} \frac{\ln ^{+} \ln ^{+}\left|b_{n}\right|}{\ln \left|a_{n}\right|+2}<\infty
$$

(C) Petrozavodsk State University, 2019 


$$
\limsup _{\left|a_{n}\right| \rightarrow \infty} \frac{\ln ^{+} \ln ^{+}\left|b_{n}\right|}{\ln \left|a_{n}\right|} \leqslant \rho,
$$

there exists a function $F \in[\rho, \infty]^{+}$that solves the interpolation problem

$$
F\left(a_{n}\right)=b_{n}, \quad n=1,2, \ldots
$$

Here $\ln ^{+} b=\left\{\begin{array}{l}0, b \leqslant 0 \\ \ln b, b>0 .\end{array}\right.$

Denote by $B_{q}(u, v)$ the Nevanlinna primary factor

$$
B_{q}(u, v)=\left\{\begin{array}{l}
\frac{\bar{v}(u-v)}{v(u-\bar{v})}, \quad q=0, \\
B_{0}(u, v) \exp \left(\sum_{i=1}^{q} \frac{u^{i}}{i}\left(\frac{1}{v^{i}}-\frac{1}{\bar{v}^{i}}\right)\right), \quad q \in \mathbb{N} .
\end{array}\right.
$$

Let $A=\left\{a_{n}=r_{n} e^{i \theta_{n}}\right\}_{n=1}^{\infty} \subset \mathbb{C}_{+}, r_{n}>\delta_{0}>0$, be a sequence of distinct complex numbers such that all limit points of $A$ are on the real axis and infinity, and for any $\varepsilon>0$

$$
\sum_{n=1}^{\infty} \frac{\sin \theta_{n}}{r_{n}^{\rho+\varepsilon}}<\infty, \quad \rho>1
$$

then the function

$$
E(z)=E_{A}(z)=: \prod_{\left|a_{n}\right|<1}\left(\frac{z-a_{n}}{z-\bar{a}_{n}}\right) \prod_{\left|a_{n}\right| \geqslant 1} B_{q}\left(z, a_{n}\right), \quad q=[\rho]
$$

belongs to the space $[\rho, \infty]^{+}$. Denote by [.] the integer part of a number. The function $E(z)$ is called the canonical function of the sequence $A$.

The following theorem was proved in [6].

Theorem A. The following two statements are equivalent:

1) The sequence $A$ is an interpolation sequence in the space $[\rho, \infty]^{+}$.

2) Condition (4) is true and the canonical function $E(z)$ of the sequence $A$ satisfies the conditions

$$
\begin{aligned}
& \sup _{n \in \mathbb{N}} \frac{1}{\ln \left|a_{n}\right|+2} \ln ^{+} \ln ^{+} \frac{1}{\left|E^{\prime}\left(a_{n}\right)\right| \operatorname{Im} a_{n}}<\infty, \\
& \limsup _{\left|a_{n}\right| \rightarrow \infty} \frac{1}{\ln \left|a_{n}\right|} \ln ^{+} \ln ^{+} \frac{1}{\left|E^{\prime}\left(a_{n}\right)\right| \operatorname{Im} a_{n}} \leqslant \rho .
\end{aligned}
$$


The aim of this paper is to obtain necessary and sufficient conditions for solvability of this interpolation problem in terms of measure determined by the sequence $A$. We need the following definitions. By $C(z, R)$ we denote the open disk of radius $R$ with centre in a point $z$.

Definition 2. An absolutely continuous function $\rho(r)$ on the half-axis $(0,+\infty)$ that satisfies the conditions

$$
\lim _{r \rightarrow+\infty} \rho(r)=\rho, \quad \lim _{r \rightarrow+\infty} \rho^{\prime}(r) r \ln r=0,
$$

is called a proximate order.

Here $\rho^{\prime}(r)$ is the maximum absolute value of the derivative numbers.

Let us denote $V(r):=r^{\rho(r)}$ and $V_{i}(r):=r^{\rho_{i}(r)}$, where $\rho_{i}(r)$ is a proximate order such that $\lim _{r \rightarrow+\infty} \rho_{i}(r)=\rho$. From the sequence $A$, we define the Nevanlinna measure $\mu(G):=\mu_{A}(G):=\sum_{a_{n} \in G} \sin \theta_{n}$ and the families of functions

$$
\widetilde{\Phi}_{z}^{+}(\alpha)=\mu\left(C(z, \alpha|z|) \backslash a_{n}\right), \quad \Phi_{z}^{+}(\alpha)=\frac{\widetilde{\Phi}_{z}^{+}(\alpha)}{V(|z|)}, \quad \alpha>0,
$$

where $a_{n}$ is the point closest to $z$ (if there are several such points, choose the one with the largest $\left.\sin \theta_{n}\right)$. Set

$$
\begin{gathered}
I^{+}(z, \delta)=\sin \theta \int_{0}^{\delta} \frac{\Phi_{z}^{+}(\alpha) d \alpha}{\alpha(\alpha+\sin \theta)^{2}}, \quad \theta=\arg z, \\
I^{+}\left(z, \delta_{1}, \delta_{2}\right):=I^{+}\left(z, \delta_{2}\right)-I^{+}\left(z, \delta_{1}\right), \quad \delta_{1} \leqslant \delta_{2} .
\end{gathered}
$$

Our main results are stated in the following two theorems.

Theorem 1. The following two statements are equivalent:

1) The sequence $A$ is an interpolation sequence in the space $[\rho, \infty]^{+}$.

2) Condition (4) is true, and for any $\delta>0$

$$
\limsup _{r \rightarrow \infty} \frac{1}{\ln r} \ln \left[\sin \theta \int_{0}^{\delta} \frac{\widetilde{\Phi}_{z}^{+}(\alpha) d \alpha}{\alpha(\alpha+\sin \theta)^{2}}\right] \leqslant \rho, \quad z=r e^{i \theta},
$$




$$
\sup _{z \in \mathbb{C}_{+}} \frac{1}{\ln (r+2)} \ln \left[\sin \theta \int_{0}^{\delta} \frac{\widetilde{\Phi}_{z}^{+}(\alpha) d \alpha}{\alpha(\alpha+\sin \theta)^{2}}\right]<\infty .
$$

Theorem 2. The following two statements are equivalent:

1) The sequence $A$ is an interpolation sequence in the space $[\rho, \infty]^{+}$.

2) Condition (4) is true, and for any $\delta>0$ there exists a proximate order $\rho(r), \lim _{r \rightarrow \infty} \rho(r)=\rho>1$, such that

$$
\begin{gathered}
\ln \left|\frac{a_{n}-\bar{a}_{k}}{a_{n}-a_{k}}\right| \leqslant V\left(r_{n}\right), \quad n \neq k, \\
\Phi_{z}^{+}(\alpha) \leqslant \alpha, \quad \frac{\sin \theta}{2} \leqslant \alpha \leqslant \delta, \\
\Phi_{z}^{+}(\alpha) \leqslant \sin \theta / \ln \frac{\sin \theta}{\alpha}, \quad 0 \leqslant \alpha \leqslant \frac{\sin \theta}{2},
\end{gathered}
$$

where $\theta=\arg z$.

2. Preliminaries. Denote by $[\rho(r), \infty)^{+}$the space of functions, analytic in the half-plane $\mathbb{C}_{+}$and of at most normal type for $\rho(r)$, i. e., such that $\ln |f(z)| \leqslant C_{f} V(|z|)$, where $C_{f}$ is a finite constant.

Definition 3. The sequence $A$ is called an interpolation sequence in the space $[\rho(r), \infty)^{+}$if for any sequence of complex numbers $\left\{b_{n}\right\}_{n=1}^{\infty}$ satisfying the condition

$$
\limsup _{\left|a_{n}\right| \rightarrow \infty} \frac{\ln ^{+}\left|b_{n}\right|}{V\left(\left|a_{n}\right|\right)}<\infty
$$

there exists a function $F \in[\rho(r), \infty)^{+}$that solves the interpolation problem (3).

The following theorem is the corollary of [5, Theorem 1, Theorem 2]. Theorem B. The following three statements are equivalent:

1) The sequence $A$ is an interpolation sequence in the space $[\rho(r), \infty)^{+}$.

2) The canonical function $E(z)$ of the sequence $A$ satisfies the condition

$$
\begin{gathered}
\sup _{n \in \mathbb{N}} \frac{1}{V\left(\left|a_{n}\right|\right)} \ln ^{+} \frac{1}{\left|E^{\prime}\left(a_{n}\right)\right| \operatorname{Im} a_{n}}<\infty, \\
\sup _{z \in \mathbb{C}_{+}} \sin \theta \int_{0}^{1 / 2} \frac{\Phi_{z}^{+}(\alpha) d \alpha}{\alpha(\alpha+\sin \theta)^{2}}<\infty, \quad \theta=\arg z .
\end{gathered}
$$


We will need the following inequality [5, p. 264]:

$$
\Phi_{z}^{+}(\alpha) \leqslant M_{0} \Phi_{a_{n}}^{+}(\alpha)
$$

for $\alpha \leqslant 1 / 2$, where $a_{n}$ is the point of $A$ closest to $z$.

3. Proof of Theorem 1. Now, we prove Theorem 1.

Proof. Let the sequence $A$ be an interpolation sequence in the space $[\rho, \infty]^{+}$. Then conditions (4), (5) and (6) are true. It follows from (5) and (6), that there exists a proximate order $\rho(r), \lim _{r \rightarrow \infty} \rho(r)=\rho>1$, such that condition (13) is true. By Theorem B, we obtain condition (14) from (13). Obviously, condition (14) implies conditions (7) and (8).

Now, let conditions (7) and (8) be true. Then there exists a proximate order $\rho_{1}(r), \lim _{r \rightarrow \infty} \rho_{1}(r)=\rho$, such that condition (14) is true. Let a sequence $\left\{b_{n}\right\}_{n=1}^{\infty}$ satisfy conditions (1) and (2). There exists a proximate order $\rho_{2}(r), \lim _{r \rightarrow \infty} \rho_{2}(r)=\rho$, such that condition (12) is true. Let $\rho(r)$, $\lim _{r \rightarrow \infty} \rho(r)=\rho$, be a proximate order such that

$$
\rho(r) \geqslant \max \left\{\rho_{1}(r), \rho_{2}(r): r>0\right\} .
$$

Conditions (12) and (14) are true by $\rho(r)$. By Theorem B, the sequence $A$ is the interpolation sequence in the space $[\rho(r), \infty)^{+}$. Therefore, there exists a function $F \in[\rho(r), \infty)^{+}$solving the interpolation problem (3). Since $[\rho(r), \infty)^{+} \subset[\rho, \infty]^{+}$for each proximate order $\rho(r)$, such that $\lim _{r \rightarrow \infty} \rho(r)=\rho$, then $F \in[\rho, \infty]^{+}$.

4. Proof of Theorem 2. Now let us prove Theorem 2.

Proof. Let conditions (9), (10) and (11) be true. Then

$$
I^{+}(z, \delta)=I^{+}\left(z, \frac{\sin \theta}{2}\right)+I^{+}\left(z, \frac{\sin \theta}{2}, \delta\right) .
$$

Using (10), we estimate the second term:

$$
\begin{gathered}
I^{+}\left(z, \frac{\sin \theta}{2}, \delta\right) \leqslant \sin \theta \int_{(\sin \theta) / 2}^{\delta} \frac{d \alpha}{(\alpha+\sin \theta)^{2}}= \\
=-\left.\frac{\sin \theta}{\alpha+\sin \theta}\right|_{(\sin \theta) / 2} ^{\delta}<\frac{2}{3} .
\end{gathered}
$$


Let us estimate the first term. To do this, we will prove

$$
\sup _{z \in \mathbb{C}_{+}} I^{+}\left(z, \frac{\sin \theta}{2}\right)<\infty
$$

From inequalities (9) and

$$
\left|\frac{a_{n}-a_{k}}{\bar{a}_{n}-a_{k}}\right| \leqslant \frac{\left|a_{n}-a_{k}\right|}{\operatorname{Im} a_{n}}
$$

we obtain $\left|a_{n}-a_{k}\right| \geqslant \operatorname{Im} a_{n} \exp \left(-M_{1} V\left(r_{n}\right)\right), n \neq k$, where $M_{1}>0$ is some constant. From this it follows that the disks $C\left(a_{n}, \operatorname{Im} a_{n} \exp \left(-M_{2} V\left(r_{n}\right)\right)\right.$ do not intersect for some $M_{2}>0$. Then

$$
\begin{gathered}
I^{+}\left(a_{n}, \frac{\sin \theta_{n}}{2}\right)=I^{+}\left(a_{n}, \sin \theta_{n} \exp \left(-M_{2} V\left(r_{n}\right)\right), \frac{\sin \theta_{n}}{2}\right) \leqslant \\
\leqslant \int_{\sin \theta_{n} / 2}\left(\ln \frac{\sin \theta_{n}}{\alpha}\right)^{-1} \frac{\sin ^{2} \theta_{n} d \alpha}{\alpha\left(\alpha+\sin \theta_{n}\right)^{2}}= \\
\quad \sin \theta_{n} \exp \left(-M_{2} V\left(r_{n}\right)\right) \\
\quad \int_{2}^{\exp \left(M_{2} V\left(r_{n}\right)\right)} \frac{\alpha d \alpha}{(\alpha+1)^{2} \ln \alpha} \leqslant \int_{2}^{\exp \left(M_{2} V\left(r_{n}\right)\right)} \frac{d \alpha}{\alpha \ln \alpha} \leqslant \\
\leqslant \ln M_{2}+\ln V\left(r_{n}\right)=\ln M_{2}+\rho\left(r_{n}\right) \ln r_{n} .
\end{gathered}
$$

Let $\rho_{1}(r), \lim _{r \rightarrow \infty} \rho_{1}(r)=\rho$, be a proximate order, such that for $r>0$ $\rho_{1}(r) \geqslant \rho(r) \ln r$. For this proximate order

$$
\sup _{n} I^{+}\left(a_{n}, \frac{\sin \theta_{n}}{2}\right)<\infty
$$

To conclude the proof of (17), it remains to see that $\Phi_{z}^{+}(\alpha) \leqslant M_{0} \Phi_{a_{n}}^{+}(\alpha)$ (see (15)) for $\alpha \leqslant 1 / 2$, where $a_{n}$ is the point of $A$ closest to $z$.

Condition (14) follows from (16) and (17).

Conversely, let conditions (7) and (8) be true. Then, for some proximate order $\rho(r), \lim _{r \rightarrow \infty} \rho(r)=\rho$, condition $(14)$ is true. Let $\beta \in[(\sin \theta) / 2, \delta]$. 
Then, for some $M_{3}>0$

$$
\begin{aligned}
& M_{3} \geqslant I^{+}(z, \beta, 2 \beta) \geqslant \sin \theta \Phi^{+}(z, \beta) \int_{\beta}^{2 \beta} \frac{d \alpha}{\alpha(\alpha+\sin \theta)^{2}} \geqslant \\
& \geqslant \frac{\Phi^{+}(z, \beta)}{2 \beta} \int_{\beta}^{2 \beta} \frac{\sin \theta d \alpha}{\alpha(\alpha+\sin \theta)^{2}}=-\left.\frac{\Phi^{+}(z, \beta) \sin \theta}{2 \beta(\alpha+\sin \theta)}\right|_{\beta} ^{2 \beta} .
\end{aligned}
$$

Since $\frac{\sin \theta}{\beta+\sin \theta} \geqslant \frac{1}{3}$ for $\beta \geqslant \sin \theta / 2$, we obtain (10).

Similarly, for $\beta \in[0, \sin \theta / 2]$

$$
\begin{aligned}
& M_{3} \geqslant I^{+}(z, \beta, 2 \beta) \geqslant I^{+}(z, \beta, \sin \theta) \geqslant \\
& \geqslant \frac{\Phi^{+}(z, \beta)}{4 \sin \theta} \int_{\beta}^{\sin \theta} \frac{d \alpha}{\alpha}=\frac{\Phi^{+}(z, \beta)}{\sin \theta} \ln \frac{\sin \theta}{\beta} .
\end{aligned}
$$

From this, we obtain (11).

5. Interpolation in the space $\mathbf{H}^{\infty}$. Let $\mathbf{H}^{\infty}$ be the space of bounded functions in $\mathbb{C}_{+}$.

Definition 4. The sequence $A$ is called an interpolation sequence in the space $\mathbf{H}^{\infty}$, if for any bounded sequence of complex numbers $\left\{b_{n}\right\}_{n=1}^{\infty}$ there exists a function $F \in \mathbf{H}^{\infty}$ that solves the interpolation problem (3).

The following theorem is the famous Carleson theorem [1].

Carleson Theorem. The following three statements are equivalent.

1) The sequence $A$ is an interpolation sequence in the space $\mathbf{H}^{\infty}$.

2) The Blaschke product $B(z)$ satisfies the condition

$$
\begin{gathered}
\inf _{n}\left\{\operatorname{Im} a_{n}\left|B^{\prime}\left(a_{n}\right)\right|\right\}>0 . \\
\inf _{n \neq k}\left|\frac{a_{n}-a_{k}}{a_{n}-\bar{a}_{k}}\right|>0
\end{gathered}
$$

and the measure $\mu(z)=\operatorname{Im} a_{n} \delta\left(z-a_{n}\right)$ is Carleson's measure, i. e., for all $x \in \mathbb{R}$ and all $h>0$

$$
\mu((x, x+h) \times(0, h)) \leqslant K h
$$


where $K>0$ is some constant independent of $x$ and $h, \delta(z)$ is the Dirac delta function.

Our result for the space $\mathbf{H}^{\infty}$ is stated in the following proposition.

Proposition. The following two statements are equivalent.

1) The sequence $A$ is an interpolation sequence in the space $\mathbf{H}^{\infty}$.

2) The following inequality is true

$$
\sup _{z \in \mathbb{C}_{+}} \sin \theta \int_{0}^{1 / 2} \frac{\widetilde{\Phi}_{z}^{+}(\alpha) d \alpha}{\alpha(\alpha+\sin \theta)^{2}}<\infty, \quad \theta=\arg z .
$$

This proposition is a corollary of Theorem B for the case $\rho(r) \equiv 0$, $r>0$.

Remark. Condition (13) is an analogue of condition (18) in the space $[\rho(r), \infty)$. Condition (14) is an analogue of condition (19) in the space $[\rho(r), \infty)$. In contrast to condition (19), which gives the boundary density of the distribution of the imaginary parts of the interpolation nodes, condition (20) gives the interior density of the distribution of the arguments of the interpolation nodes.

Acknowledgment. The authors are thankful to the referee for valuable suggestions towards the improvement of the paper.

The reported study was funded by RFBR according to the research project No 18-01-00236.

\section{References}

[1] Carleson L. An interpolation problem for bounded analytic functions. Amer. J. Math., 1958, vol. 80, pp. 921-930.

[2] Govorov N. V. Riemann's boundary problem with infinite index. Basel; Boston; Berlin: Birkhäuser. 1994.

[3] Grishin A. F. On regularity of the growth of subharmonic functions. Teor. Funktsii Funktsional. Anal. i Prilozhen., 1968, vol. 7, pp. 59-84 (in Russian).

[4] Levin B. Ya. Distribution of Zeros of Entire Functions. English revised edition Amer. Math. Soc.: Providence, RI. 1980.

[5] Malyutin K. G. The problem of multiple interpolation in the half-plane in the class of analytic functions of finite order and normal type. Russian Acad. Sci. Sb. Math., 1994, vol. 78, no. 1, pp. 253-266. 
[6] Malyutin K. G., Gusev A. L. The interpolation problem in the spaces of analytical functions of finite order in the half-plane. Probl. Anal. Issues Anal., Special Issue, 2018, vol. 7 (25), pp. 113-123.

DOI: https://doi.org/10.15393/j3.art.2018.5170

Received July 11, 2019.

In revised form, November 3, 2019.

Accepted November 3, 2019.

Published online November 9, 2019.

K. G. Malyutin

Kursk State University

33 Radischeva str., Kursk 305000, Russia

E-mail: malyutinkg@gmail.com

A. L. Gusev

Kursk State University

33 Radischeva str., Kursk 305000, Russia

E-mail: cmex1990goose@yandex.ru 